\title{
Highly Efficient Quantum Dot Near-Infrared Light-Emitting Diodes
}

\author{
Xiwen Gong, Zhenyu Yang, Grant Walters, Riccardo Comin, \\ Zhijun Ning, Eric Beauregard, Valerio Adinolfi, \\ Oleksandr Voznyy, and Edward H. Sargent
}

\begin{abstract}
Version Post-Print/Accepted Manuscript
Citation Gong, X., Yang, Z., Walters, G., Comin, R., Ning, Z., Beauregard, E., (published version) Adinolfi, V., Voznyy, O., and Sargent, E. H. (2016). Highly efficient quantum dot near-infrared light-emitting diodes. Nature Photonics, 10(4), 253-257. doi:10.1038/nphoton.2016.11
\end{abstract}

Publisher's Statement The final published version of this paper is available at Nature Publishing Group via http://dx.doi.org/10.1038/nphoton.2016.11.

\section{How to cite TSpace items}

Always cite the published version, so the author(s) will receive recognition through services that track citation counts, e.g. Scopus. If you need to cite the page number of the TSpace version (original manuscript or accepted manuscript) because you cannot access the published version, then cite the TSpace version in addition to the published version using the permanent URI (handle) found on the record page. 


\title{
Highly Efficient Quantum Dot Near-Infrared Light-Emitting Diodes
}

\author{
Xiwen Gong ${ }^{1}$, Zhenyu Yang ${ }^{1}$, Grant Walters, Riccardo Comin, Zhijun Ning ${ }^{\dagger}$, Eric Beauregard, \\ Valerio Adinolfi, Oleksandr Voznyy, and Edward H. Sargent* \\ Department of Electrical and Computer Engineering, University of Toronto, 10 King's College \\ Road. Toronto, Ontario, M5S 3G4, Canada \\ E-mail: ted.sargent@utoronto.ca \\ 1 These authors contribute equally to this work \\ $\dagger$ Present address: School of Physical Science and Technology, ShanghaiTech University, Haike \\ Road 100, 201210 Shanghai, China.
}

Colloidal quantum dots (CQDs) are emerging as promising materials for constructing infrared sources in view of their tunable luminescence, high quantum efficiency and compatibility with solution processing. However, CQD films today suffer a compromise between luminescence efficiency and charge transport, and this leads to unacceptably high power consumption. Here we overcome this issue by embedding CQDs in a high-mobility hybrid perovskite matrix. The new composite enhances radiative recombination in the dots by preventing transport-assisted trapping losses; yet does so without increase the turn-on voltage. Through compositional engineering of the mixed halide matrix, we achieve a record electroluminescence power conversion efficiency of $4.9 \%$. This surpasses the performance of previously-reported CQD near-infrared devices by two-fold, indicating great promise for this hybrid QD in perovskite approach. 
Near-infrared (NIR) light-emitting diodes (LEDs) are used in a wide range of applications including night vision ${ }^{1}$, biomedical imaging ${ }^{2}$, and optical communications and computing ${ }^{3}$. While advanced materials such as organic dyes and semiconductor polymers have shown great benefits in visible lighting ${ }^{4,5}$, they have so far failed to produce efficient NIR LEDs because of their large bandgap; to the extent that their bandedge can be redshifted, this consistently results in a low photoluminescence quantum yield in the NIR regime ${ }^{6}$. In light of CQDs' high luminescence efficiency, emission wavelength tunability, and solution processability, researchers have invested considerable efforts exploring NIR emitting devices based on these materials ${ }^{7}$, and reported power conversion efficiencies (PCE) have recently reached $2.4 \%{ }^{8}$.

PCE is defined as the ratio of the output optical power $\left(P_{\text {out }}\right)$ to the input electrical power $\left(P_{\text {in }}\right)$. Combined with the definition of external quantum efficiency (EQE) ${ }^{6}$, the PCE can be written:

$$
\begin{aligned}
& P C E=\frac{P_{\text {out }}}{P_{\text {in }}}=\frac{P_{\text {out }}}{I V}=\frac{E Q E \cdot h v}{\mathrm{eV}}=\frac{\eta_{\text {tot }} \cdot \eta_{\text {out }} \cdot h v}{\mathrm{eV}} \\
& \eta_{\text {tot }}=\frac{n_{\text {charges }}}{n_{\text {photons }}}=\eta_{\text {diff }} \cdot \eta_{\text {inj }} \cdot \chi \cdot \eta_{P L Q E}
\end{aligned}
$$

where $\eta_{\text {tot }}$ is the fraction of injected charges $\left(n_{\text {charges }}\right)$ that produce the emission of photons from the CQDs; $\eta_{\text {out }}$ is the fraction of emitted photons $\left(n_{\text {photons }}\right)$ that are coupled out of the device; $h v$ is the energy of the emitted photons; $e$ is the elementary charge; and $V$ is the voltage applied to the device. $\eta_{\text {diff }}$ is the fraction of injected carriers that successfully diffuse to a quantum dot; $\eta_{i n j}$ is the fraction of these carriers that transfer into a quantum dot and form excitons inside; $\chi$ is the fraction of these excitons whose states have spin-allowed optical 
transitions (for CQDs, $\chi=1$ ); $\eta_{P L Q E}$ is the internal CQDs photoluminescence quantum efficiency (PLQE) associated with radiative emission from a CQD-localized exciton.

Clearly, high total luminescence efficiency $\eta_{\text {tot }}$ and low operating voltage $V$ are key parameters for high PCE. Unfortunately, luminescence efficiencies of CQD films, especially for NIR $\mathrm{CQDs}^{9}$, are generally very low. This is the result of self-quenching, wherein inter-dot transport facilitates transport-assisted trapping, leading to recombination even at relatively rare defect sites $^{10}$. Materials with highly mobile carriers, such as close-packed CQD films, can also exhibit exciton dissociation, and this competes with the radiative recombination and further enhances self-quenching ${ }^{11}$.

Strategies to prevent self-quenching in CQD films typically include the growth of a protective shel1 ${ }^{12}$, capping with insulating organic ligands ${ }^{9}$, and incorporation into a polymer matrix ${ }^{9,13,14}$. However, these solutions lead to increased power consumption since a higher voltage is required to inject sufficient current to achieve bright light emission. This trade-off between non-radiative recombination and charge transport has persisted, and accounts for the modest PCEs of CQDbased LEDs.

We took the view that quantum dots combined with perovskite ${ }^{15,16}$ could potentially overcome the trade-off between quantum efficiency and voltage efficiency: the perovskite matrix would provide a long diffusion length to enhance charge carriers' diffusion efficiency $\left(\eta_{\text {diff }}\right)$, even when CQDs epitaxially embedded inside matrix were widely spaced with the goal of avoiding self-quenching and exciton dissociation (Fig.1a). The epitaxial interface ${ }^{15}$ between the perovskite matrix and the CQDs would serve to provide excellent passivation, improving both $\eta_{i n j}$ and $\eta_{P L Q E}$. The use of a type-I band alignment would enable electrons and holes to be funneled 
efficiently from the matrix and be confined inside the CQDs, again preventing exciton dissociation losses.

Prior works on quantum dot:perovskite solids suffered from a lattice mismatch $(\sim 5 \%)$ between iodide-based perovskite and $\mathrm{PbS}$ nanocrystals. We explored variations in the perovskite lattice constant via mixing of iodide and bromide (Fig. 1a, 1b). The PLQE of quantum dot inclusions and the carrier transport in the matrix are greatly increased in the mixed halide perovskite matrix $\left(\mathrm{MAPbI}_{\mathrm{x}} \mathrm{Br}_{3-\mathrm{x}}\right)$ compared to the $1^{\text {st }}$ generation quantum-dot-in-perovskites that relied on $\mathrm{MAPbI}_{3}{ }^{18}$. The introduction of bromide also enables a pinhole-free morphology and improves the carrier diffusion length in the perovskite matrix, resulting in lower trap-assisted recombination and consequently in more efficient emission from the active layer.

Our LED device architecture is described in Fig. 1b: indium tin oxide (ITO) / titanium dioxide $\left(\mathrm{TiO}_{2}, 10 \mathrm{~nm}\right) /$ dots-in-perovskite emission layer (350 nm) (Fig. 1c) / poly(9,9'-dioctylfluorene) $(\mathrm{F} 8,50 \mathrm{~nm}) /$ molybdenum trioxide $\left(\mathrm{MoO}_{3}, 6 \mathrm{~nm}\right) /$ silver $(\mathrm{Ag}, 100 \mathrm{~nm})$. As seen from the calculated band diagram for the LED device at zero bias (Fig. S1, top panel), the energy levels of F8 and $\mathrm{TiO}_{2}$ layers are suitably aligned with the perovskite matrix to serve as electron and hole blocking layers, respectively, while enabling selective injection of the desired carrier type into the active layer. In addition, a type-I heterojunction between the quantum dot acceptor levels (dashed black line) and perovskite bandedges (blue and red line for the conduction and valence band, respectively) is maintained throughout the emissive QD-in-perovskite layer. Under forward bias (Fig. S1, bottom panel) electrons and holes are injected from the cathode $\left(\mathrm{TiO}_{2}\right)$ and anode (F8), respectively, and they are efficiently transferred to the quantum dots where they recombine radiatively. 
The EQE curves (Fig. 2a) highlight the efficiency increase upon incorporation of small amounts of bromine, with a maximum EQE value of $3.8 \%$ for a matrix with $83 \%$ iodine molar concentration (the molar ratio between iodine and all halides). The EQE is 2.3 times higher than for the pure $\mathrm{MAPbI}_{3}$ matrix. However, EQE drops with lower iodine content, with no NIR light emission for iodide molar concentrations below $67 \%$. To investigate the origins of the matrixcomposition-dependent electroluminescence of CQDs, we further investigated the photophysical properties of quantum dots in the $\mathrm{MAPbI}_{x} \mathrm{Br}_{3-\mathrm{x}}$ matrix.

The first important function of the perovskite matrix is to transport photogenerated carriers into the quantum dots. We found that the diffusion length (Fig. 2b), which will determine the efficiency with which electron-hole pairs can be delivered to the CQDs, shows a significant enhancement once bromine is incorporated into the matrix. ${ }^{17}$ The diffusion length and the photoluminescence lifetime (Fig. S2) both peak at $83 \%$ iodine halide content. This perovskite matrix also shows the best stability in moist air (Fig. S3).

We then studied the quality of the interface between quantum dots and perovskite. Fig. $2 \mathrm{c}$ shows that the PLQE of CQDs photoexcited below the perovskite bandgap increases with higher bromine concentration and peaks at $50 \%$ iodine concentration. As in previous work ${ }^{15}$, the perovskite matrix epitaxially grows around the quantum dots, providing a natural passivation of the CQD surface, and this leads to higher $\eta_{P L Q E}$ and $\eta_{i n j}$. We propose that the furtherimproved lattice matching reported herein through the use of the mixed halide matrix reduces strain at the heteroepitaxial interface, and further lowers the density of defects. We measured powder x-ray diffraction of pure quantum dots and in the $\mathrm{MAPbI}_{x} \mathrm{Br}_{3-\mathrm{x}}$ perovskite matrix to evaluate the lattice mismatch (Fig. S4). Since the PbS lattice constant (5.91 Å) lies between that of $\mathrm{MAPbI}_{3}(6.26 \AA)$ and $\mathrm{MAPbBr}_{3}(5.89 \AA)$, the lattice matching improves with increasing 
bromine concentration and peaks at $67 \%$ iodine content, where the interface exhibits the lowest strain.

In sum, the addition of bromine reduces the defect density both inside the perovskite matrix and on the CQD surface, improving diffusion efficiency and PLQE. Reduced non-radiative recombination losses should result in higher total luminescence efficiency of the material. Our optoelectronic device modeling shows that trap recombination is the most detrimental mechanism at low driving voltages (low brightness regime) while, at higher injection densities, Auger recombination becomes the dominant loss. Simulations in Fig. $2 \mathrm{~d}$ show that, across the entire range of achievable LED brightnesses, devices with the lowest trap density give the highest $\eta_{t o t}$, highlighting the importance of controlling trap-related losses inside the conductive active layer.

When CQDs are evenly distributed inside the perovskite matrix at low concentrations, the electroluminescence (EL) properties are dramatically improved compared with pure CQD-based devices. The strongest EL intensity observed in this work was from devices emitting at $1391 \mathrm{~nm}$. The blueshift in the EL signal from CQD-in-perovskite devices compared to CQD-only counterparts is consistent with the shift in PL (Fig. 3a and S5). We attribute the EL enhancement to the reduced self-quenching and excellent passivation from perovskite, especially at low CQD concentration at which dots are well-separated from one other (Fig. 4a). The best EQE is attained at 3.6\% CQD concentration. The best devices emit at $1391 \mathrm{~nm}$ with an EQE of $5.2 \pm 0.3 \%$ (Fig. 3b). The average value for all measured devices is $4.7 \pm 0.4 \%$ (Fig. 3f), comparable to the average for previously-reported core/shell $\mathrm{PbS} / \mathrm{CdS}$ CQD-based LEDs ${ }^{8}$. The EQE value is 150 times higher than that of a CQD-only control device $(\mathrm{EQE}=0.03 \pm 0.01 \%)$. 
Compared with other matrix materials such as polymers and inorganic crystals, the best perovskites exhibit considerably lower trap densities and higher mobility ${ }^{18}$. This will beneficially affect the driving voltage required to achieve high brightness and thus the power conversion efficiency (Fig. S6c), a figure of particular relevance to practical device operation. To study how EQE translates into PCE, we investigated the current-voltage $(J-V)$ characteristics in greater detail (Fig. 3c). The higher current density observed in the pure CQD device is due to the smaller bandgap (CQD: $0.89 \mathrm{eV}$ vs. perovskite matrix: $1.73 \mathrm{eV}$ ) and a reduced thickness of the active layer. In the quantum-dot-in-perovskite devices, the current density is considerably lower at low applied voltages, producing a higher EQE. We attribute this, once again, to the epitaxiallyaligned CQD/perovskite interface which passivates CQD trap states and reduces non-radiative recombination.

We determined a turn-on voltage of $1.0 \mathrm{~V}$ for the matrix-based LEDs (Fig. 3d), indicating effective carrier injection from the charge transport layer to the CQD inclusions. The brightness at each voltage is dramatically improved. For example, we measured a peak radiance of $2.6 \mathrm{Wsr}^{-1} \mathrm{~m}^{-2}$ at $3.5 \mathrm{~V}$ applied voltage, which is $\sim 15$ times higher than the control pure CQD device (Fig. S7). A similar increase in radiance is also found in matrix-based devices with smaller embedded dots emitting at $1250 \mathrm{~nm}$ (Fig. S8).

The combination of relatively high radiance and absolute output power, together with high EQE with low driving voltage, allows notable improvements in PCE values relative to prior reports (Fig. 3e). A peak PCE of $4.9 \%$ is obtained at $1.1 \mathrm{~V}$; more than two times higher than previous record values for IR CQD EL (Table S1) ${ }^{8}$. The PCE values exceed $1 \%$ in the full range $1-2 \mathrm{~V}$, beyond which they maintain high radiance (up to $0.92 \mathrm{~W} \mathrm{sr}^{-1} \mathrm{~m}^{-2}$ ). 
In sum, the perovskite-matrix-based devices simultaneously achieve both high radiance and efficiency (Fig. 3f). The device radiance at maximum PCE is $\sim 3.5 \mathrm{x}$ higher than in previous record CQD IR EL devices ${ }^{8}$; similarly, the PCE at maximum radiance is also improved.

We showcased the same architecture and physical principles using a number of different CQDs sizes. Consistent with the PLQE results (Fig. S9), both the peak EQE and PCE average values increase as the CQD size increases (Fig. 4). Although there is some variance at lower CQD concentrations, the EQE and PCE values are both maximized in devices with low CQDs loadings (3.6\% volume ratio). In particular, the EQE values decrease significantly at higher CQD concentrations (e.g., 16\% volume ratio), a behavior we ascribe to the imperfect passivation by the perovskite matrix and the formation of dot-to-dot intercommunication channels. However, regardless of the perovskite/CQD ratio and emission wavelength, all CQD-in-perovskite LEDs exhibited higher EQE and PCE values than those based on pure CQDs as emissive layers, suggesting that the type-I architecture is still beneficial even at high CQD loadings.

In conclusion, we have developed an original platform for bright NIR-LEDs. The work builds on a recent report of quantum-dot-in-perovskite material and significantly augments these recent findings by creating an efficient-EL-compatible matrix. Thanks to the excellent diffusion length of the iodide/bromide mixed perovskite, as well as its type-I band alignment, charge carriers are transported through the perovskite matrix to efficiently recombine in CQDs. This yields devices with a tunable emission wavelength, bandedge derived turn-on voltages, and record power efficiencies. These results highlight the potential of these emerging materials power-efficient, low-cost, and large-area LEDs and displays.

\section{References:}


1. Sun, L. et al. Bright infrared quantum-dot light-emitting diodes through inter-dot spacing control. Nature Nanotech. 7, 369-373 (2012).

2. Medintz, I. L., Uyeda, H. T., Goldman, E. R. \& Mattoussi, H. Quantum dot bioconjugates for imaging, labelling and sensing. Nature Mater. 4, 435-46 (2005).

3. Sargent, E. H. Infrared quantum dots. Adv. Mater. 17, 515-522 (2005).

4. Kim, K.-H. et al. Phosphorescent dye-based supramolecules for high-efficiency organic light-emitting diodes. Nature Commun. 5, 4769 (2014).

5. Sekine, C., Tsubata, Y., Yamada, T., Kitano, M. \& Doi, S. Recent progress of high performance polymer OLED and OPV materials for organic printed electronics. Sci. Technol. Adv. Mater. 15, 034203 (2014).

6. Shirasaki, Y., Supran, G. J., Bawendi, M. G. \& Bulović, V. Emergence of colloidal quantum-dot light-emitting technologies. Nature Photon. 7, 13-23 (2013).

7. Bourdakos, K. N., Dissanayake, D. M. N. M., Lutz, T., Silva, S. R. P. \& Curry, R. J. Highly efficient near-infrared hybrid organic-inorganic nanocrystal electroluminescence device. Appl. Phys. Lett. 92, 90-93 (2008).

8. Supran, G. J. et al. High-Performance Shortwave-Infrared Light-Emitting Devices Using Core-Shell (PbS-CdS) Colloidal Quantum Dots. Adv. Mater. 27, 1437-1442 (2015)

9. Tessler, N., Medvedev, V., Kazes, M., Kan, S. \& Banin, U. Efficient near-infrared polymer nanocrystal light-emitting diodes. Science 295, 1506-1508 (2002).

10. Zhitomirsky, D., Voznyy, O., Hoogland, S. \& Sargent, E. H. Measuring charge carrier diffusion in coupled colloidal quantum dot solids. ACS Nano 7, 5282-5290 (2013).

11. Choi, J. J. et al. Photogenerated exciton dissociation in highly coupled lead salt nanocrystal assemblies. Nano Lett. 10, 1805-1811 (2010).

12. Moroz, P. et al. Infrared emitting PbS nanocrystal solids through matrix encapsulation. Chem. Mater. 26, 4256-4264 (2014).

13. Greenham, N., Peng, X. \& Alivisatos, A. Charge separation and transport in conjugatedpolymer/semiconductor-nanocrystal composites studied by photoluminescence quenching and photoconductivity. Phys. Rev. B 54, 17628-17637 (1996).

14. Steckel, J. S., Coe-Sullivan, S., Bulović, V. \& Bawendi, M. G. $1.3 \mu \mathrm{m}$ to $1.55 \mu \mathrm{m}$ Tunable Electroluminescence from PbSe Quantum Dots Embedded within an Organic Device. Adv. Mater. 15, 1862-1866 (2003).

15. Ning, Z. et al. Quantum-dot-in-perovskite solids. Nature 523, 324-328 (2015). 
16. Hu, L. et al. PbS colloidal quantum dots as an effective hole transporter for planar heterojunction perovskite solar cells. J. Mater. Chem. A 3, 515-518 (2015).

17. Jeon, N. J. et al. Compositional engineering of perovskite materials for high-performance solar cells. Nature 517, 476-480 (2015).

18. Shi, D. et al. Low trap-state density and long carrier diffusion in organolead trihalide perovskite single crystals. Science 347, 519-522 (2015).

\section{Acknowledgements}

This publication is based in part on work supported by an award (KUS-11-009-21) from the King Abdullah University of Science and Technology (KAUST), by the Ontario Research Fund Research Excellence Program, and by the Natural Sciences and Engineering Research Council (NSERC) of Canada. X. G. thanks Mitacs for Globalink Graduate Fellowship Aeward. The authors thank L. Levina for the assistance in CQDs synthesis; X. Lan, E. Yassitepe and F. Fan for acquiring microscopic images; and E. Palmiano, R. Wolowiec, and D. Kopilovic for their help during the course of study.

\section{Author contributions}

X.G., Z.Y., Z.N., and E.H.S. designed and directed this study. X.G. and Z.Y. contributed to all the experimental work. G.W. and E.B. carried out the PLQE measurements and analysis. R.C. performed PL decay measurement and analysis. V.A. performed optoelectronic simulation. X.G., Z.Y., R.C., and E.H.S. wrote the manuscript.

\section{Additional Information}


Supplementary information is available online at www.nature.com. Reprints and permissions information is available online at www.nature.com/reprints. Correspondence and requests for materials should be addressed to E.H.S.

\section{Competing financial interests}

The authors declare no competing financial interests. 
Figure 1 | Quantum-dot-in-perovskite LED devices architecture. (a) Illustration of enhanced electroluminescence efficiency in $\mathrm{PbS}$ quantum dot in $\mathrm{MAPbX}_{3}(\mathrm{X}=\mathrm{I}, \mathrm{Br})$ perovskite CQD LEDs: left panel illustrates radiative recombination dominates when quantum dots and perovskite are lattice matched; while lattice mismatch causes interfacial defects (black dash line) and non-radiative recombination through traps (right panel). (b) The corresponding spatial band diagram schematizes the mechanism of carrier transport, injection and recombination, illustrating key parameters essential for highly efficient quantum dot LED. (c) HRTEM images of a PbS quantum dot in perovskite matrix; LED device architecture used in this study; and corresponding crossed-section SEM image of same device.

Figure 2 | Device performance and photoluminescent properties of CQDs in $\mathrm{MAPbI}_{x} \mathrm{Br}_{3-\mathrm{x}}$ perovskite. (a) EQE-current density performance of CQDs in mixed halide perovskite LEDs with various iodine molar concentrations. (b) Normalized diffusion length of the mixed halide perovskite. (c) Photoluminescence quantum efficiency of $\mathrm{PbS}$ quantum dots in $\mathrm{MAPbI}_{\mathrm{x}} \mathrm{Br}_{3-\mathrm{x}}$. (d) Normalized total efficiency $\left(\eta_{t o t}\right)$ at different radiative recombination rates $\left(\mathrm{R}_{\mathrm{rad}}\right)$ with different defect density of the active layer.

Figure 3 | Electroluminescent performance of quantum-dot-in-perovskite LEDs emitting at 1391 nm. (a) EL spectra (applied voltage: 2V), (b) EQE-current density performance, (c) current density-voltage curves, and (d) radiance-voltage characteristics; inset: zoom-in region of radiance-voltage plots $(0.9-1.3$ V) showing device turn-on voltages. (e) PCE-voltage performance for champion CQD-in-perovskite devices (3.6\% volume ratio) matrix vs. pure CQD device and (f) PCE-radiance performance of champion device compared with the current published record ${ }^{8}$ (Solid star: radiance under PCE maximum; hollow star: PCE under radiance maximum in the reference). Inset: zoom-in region of radiance-PCE plot. 
Figure 4 | Size tunability of CQDs in NIR LED. Average peak (a) EQE and (b) PCE values of dot-in-perovskite devices with various CQD:perovskite volume ratios and device emission wavelength. 


\section{Methods}

\section{CQD Synthesis and Solution Exchange}

Colloidal quantum dots (CQDs) are synthesized using methods previously reported. ${ }^{19}$ For the iodide ligand exchange, $3 \mathrm{~mL}$ of CQDs dispersed in octane $(10 \mathrm{mg} / \mathrm{mL})$ are added to a $3 \mathrm{~mL}$ dimethylformamide (DMF) solution containing $695 \mathrm{mg}$ of $\mathrm{PbI}_{2}$ and $240 \mathrm{mg}$ of $\mathrm{CH}_{3} \mathrm{NH}_{3} \mathrm{I}$. After the CQDs are stirred vigorously for 15 minutes, they transfer from the top octane phase to the bottom DMF layer.

The octane layer is then decanted and the CQD solution is washed three more times with octane to remove organic residue. Subsequently, the CQDs are precipitated by the addition of toluene. The sample is centrifuged and the solution decanted. The CQDs are dried for 10 minutes under vacuum and weighed. The nanoparticles are dispersed in butylamine for film fabrication.

\section{Quantum-Dot-in-Perovskite Solids Fabrication}

The desired amount of perovskite precursor $\left(\mathrm{PbI}_{2}\right.$ and $\mathrm{PbBr}_{2}$ with one quarter weight ratio of $\left.\mathrm{CH}_{3} \mathrm{NH}_{3} \mathrm{I}\right)$ in butylamine is added to the exchanged CQDs. The solution is spin-coated (6000 $\mathrm{rpm}, 10$ seconds) onto the substrate, and then annealed at $70^{\circ} \mathrm{C}$ for 10 minutes in an $\mathrm{N}_{2}$ glovebox to remove butylamine completely from the film ${ }^{15}$. For perovskite growth, methylammonium halide mixed solution $(10 \mathrm{mg} / \mathrm{mL}$ in isopropanol) is drop-cast on the film and removed after 30 seconds by spin coating (6000 rpm, 10 seconds), after which the film is soaked in pure isopropanol for 10 seconds, and the substrate is again spun (6000 rpm, 10 seconds) for complete removal of the residual solvent. The dots-in-perovskite film is annealed again at $70^{\circ} \mathrm{C}$ for 10 minutes in an $\mathrm{N}_{2}$ glovebox.

Fabrication of Light-Emitting Diodes 
Pre-patterned ITO-coated glass substrates are treated using oxygen plasma for 10 minutes immediately prior to use. $\mathrm{The}^{\mathrm{TiO}_{2}}$ layer is fabricated by a two-step method: (i) the initial layer is deposited at $150^{\circ} \mathrm{C}$ using atomic layer deposition (ALD) (Cambridge Savannah S100 ALD system) using tetrakis (dimethylamido) titanium (IV) and water as precursors; (ii) the film is further treated by $\mathrm{TiCl}_{4}$ aqueous solutions $(40 \mathrm{mM})$ at $70^{\circ} \mathrm{C}$ for 30 minutes. This is followed by washing with DI water, drying by $\mathrm{N}_{2}$ flow, and finally, annealing at $400^{\circ} \mathrm{C}$ for 1 hour. Once cool, the substrates are stored in a $\mathrm{N}_{2}$ glovebox for further use. The F8/chlorobenzene solution (10 $\mathrm{mg} / \mathrm{mL}$ ) is annealed at $90^{\circ} \mathrm{C}$ under nitrogen atmosphere and spin-cast onto the quantum-dot-inperovskite layer at $70^{\circ} \mathrm{C}$ and $3000 \mathrm{rpm}$ for 60 seconds to form a uniform hole transport layer. The top electrode, consisting of $6 \mathrm{~nm}$ of $\mathrm{MoO}_{3}$ and $100 \mathrm{~nm}$ of $\mathrm{Ag}$, is deposited by thermal evaporation at a pressure $<1 \times 10^{-6}$ Torr. Each ITO substrate $(2.5 \mathrm{~cm} \times 2.5 \mathrm{~cm})$ is patterned to yield eight devices, each with an area of $3.1 \mathrm{~mm}^{2}$.

\section{PL and EL Measurement}

Photoluminescence measurements are performed using a Horiba Fluorolog Time Correlated Single Photon Counting system with photomultiplier tube detectors. A monochromatized Xe lamp and pulsed laser diodes were used as excitation sources for steady-state and transient measurements, respectively. Absolute PLQE measurements were carried out in a Quanta-Phi integrating sphere according to standard methods published elsewhere ${ }^{20}$. The diffusion length of perovskite matrix was obtained by measuring PL decay with a reported quenching method, and subsequently fitting the dynamics with a 1-D diffusion equation ${ }^{21}$. For EL measurement, devices are characterized using a Keithley 2410 source meter. Under a range of forward bias voltages, the EL spectra are collected through a set of lenses focused on an optical fiber and connected to the NIR spectrophotometer (Ocean Optics, NIR-512). 


\section{Device Performance Characterization}

The radiated power of EL was measured using a calibrated Ophir PD300-IR germanium photodiode (active area: $19.6 \mathrm{~mm}^{2}$ ) and recorded in-situ using a computer-controlled Keithley 2400 source meter under nitrogen atmosphere and in parallel with the current-voltage $(I-V)$ measurements. Lambertian emission was assumed in the calculation of EQE and radiance. Peak EQE was determined as the number of forward-emitted photons to the number of injected electrons. A geometric correction factor was used according to the distance between photodiode and device and the active area of the detector. PCE was calculated as the radiative EL power divided by the total input electrical power. For each type of sample (e.g., specific quantum dot vs. perovskite concentration and size of the quantum dot), at least five devices were tested for each EQE and PCE calculations.

\section{Optoelectronic Simulation}

Device simulation was performed in SCAPS 3. 0.01 software $^{22}$. Ohmic boundary conditions were assumed on each side. Material parameters were based on data previously used in CQD solar cell simulations ${ }^{23}$. Trap density, was chosen as $10^{13}, 10^{14}$ and $10^{15} \mathrm{~cm}^{-3}$.

\section{References}

19. Hines, M. A. \& Scholes, G. D. Colloidal PbS nanocrystals with size-tunable near-Infrared emission: observation of post-synthesis self-narrowing of the particle size distribution. Adv.

Mater. 15, 1844-1849 (2003).

20. De Mello, J. C., Wittmannn, H. F. \& Friend, R. H. An improved experimental determination of external photoluminescence quantum efficiency. Adv. Mater. 9, 230 (1997).

21. Xing, G. et al. Long-range balanced electron- and hole-transport lengths in organicinorganic CH3NH3PbI3. Science 342, 344-7 (2013).

22. Burgelman, M., Nollet, P. \& Degrave, S. Modelling polycrystalline semiconductor solar cells. Thin Solid Films 527, 361-362 (2000). 
23. Zhitomirsky, D et al. Engineering colloidal quantum dot solids within and beyond the mobility-invariant regime. Nature Commun. 5, 3803 (2014). 

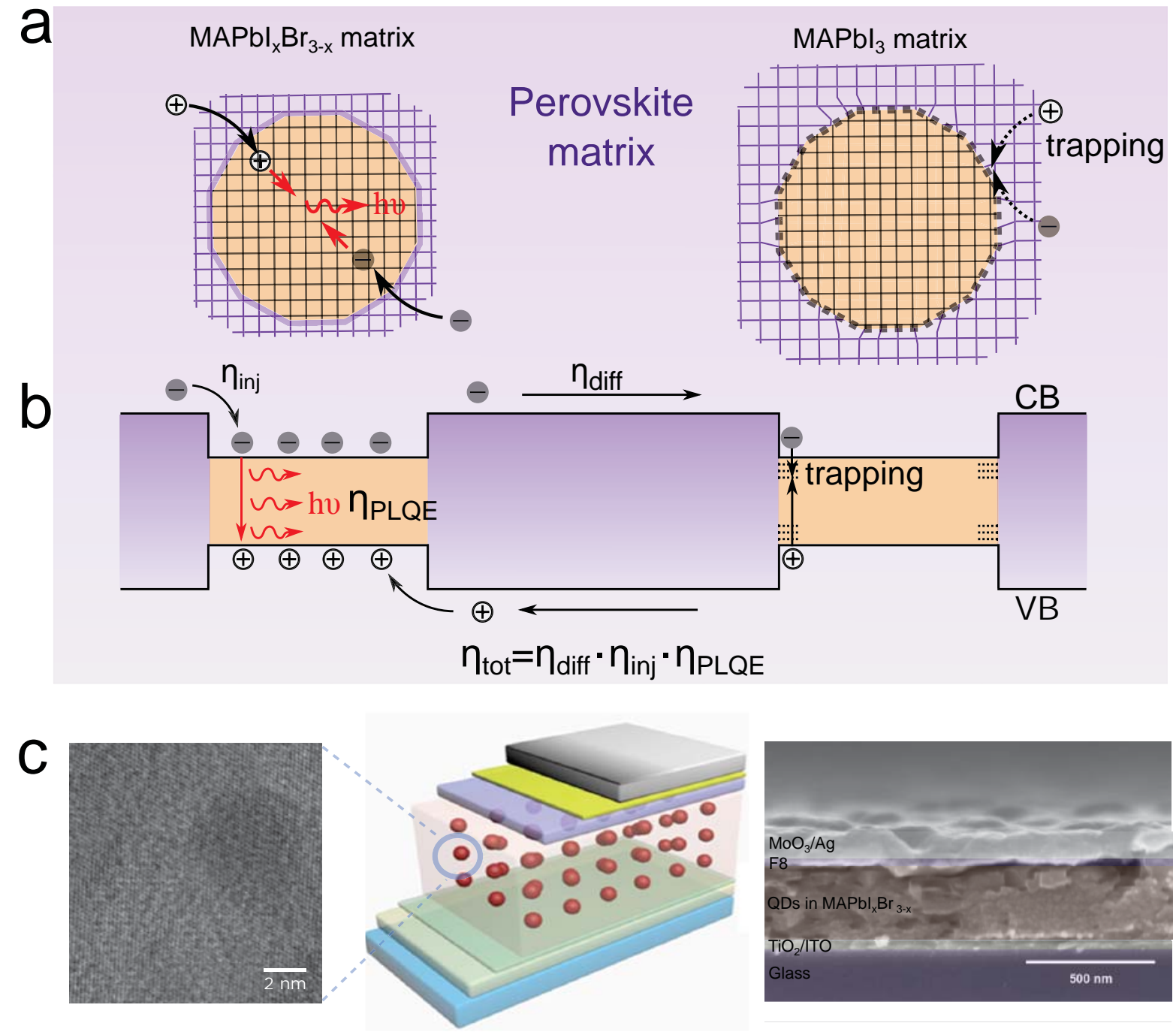

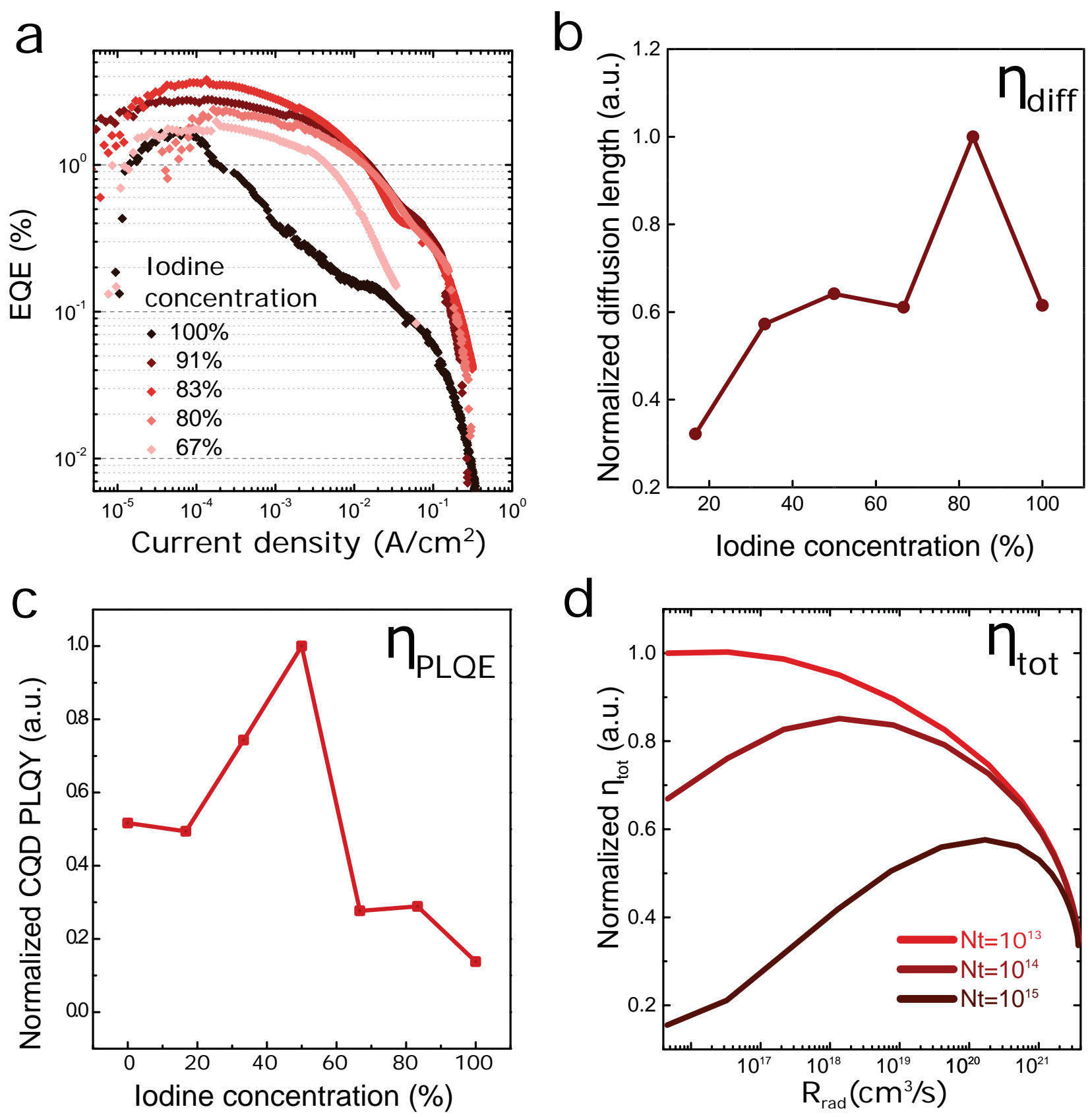

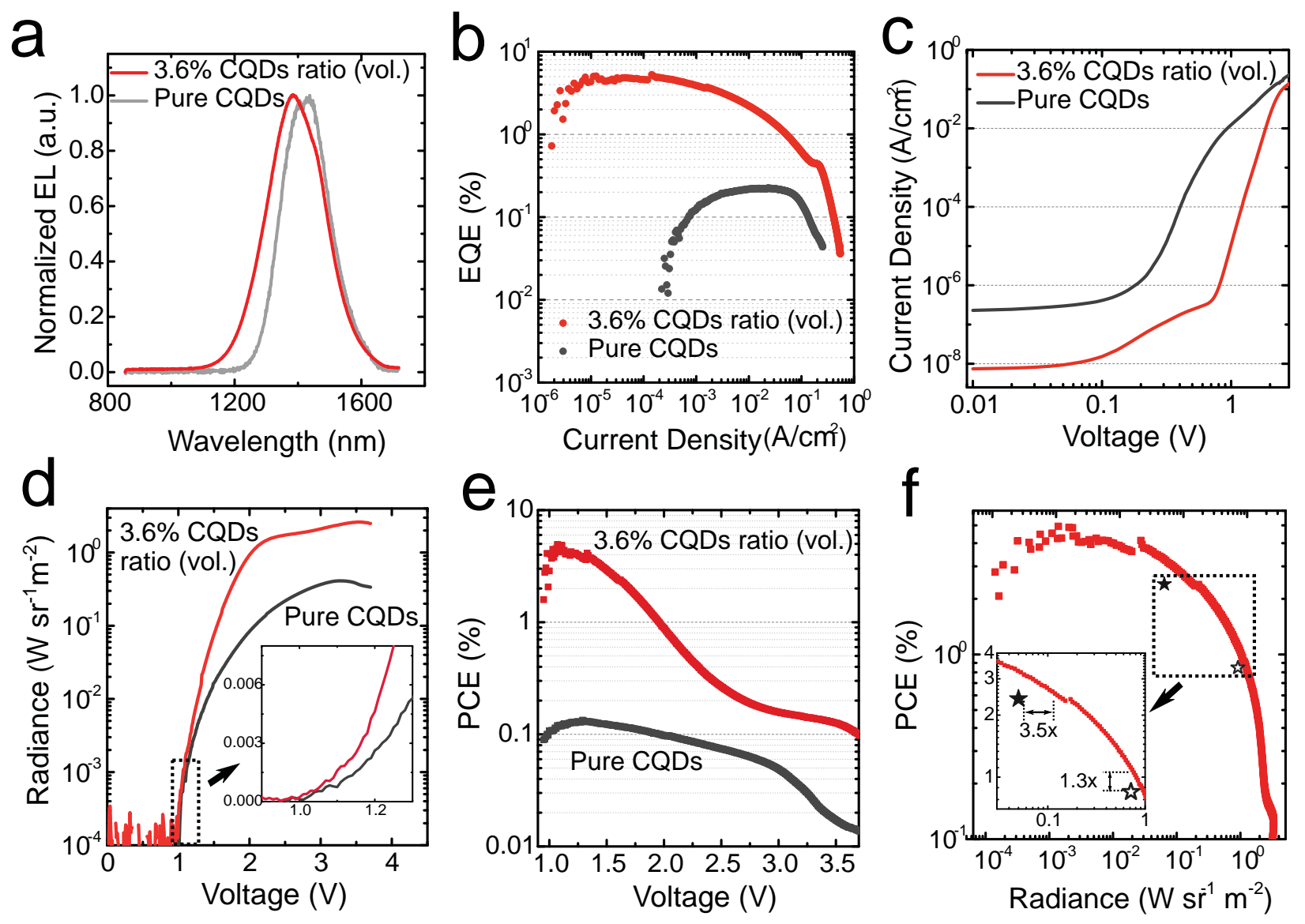

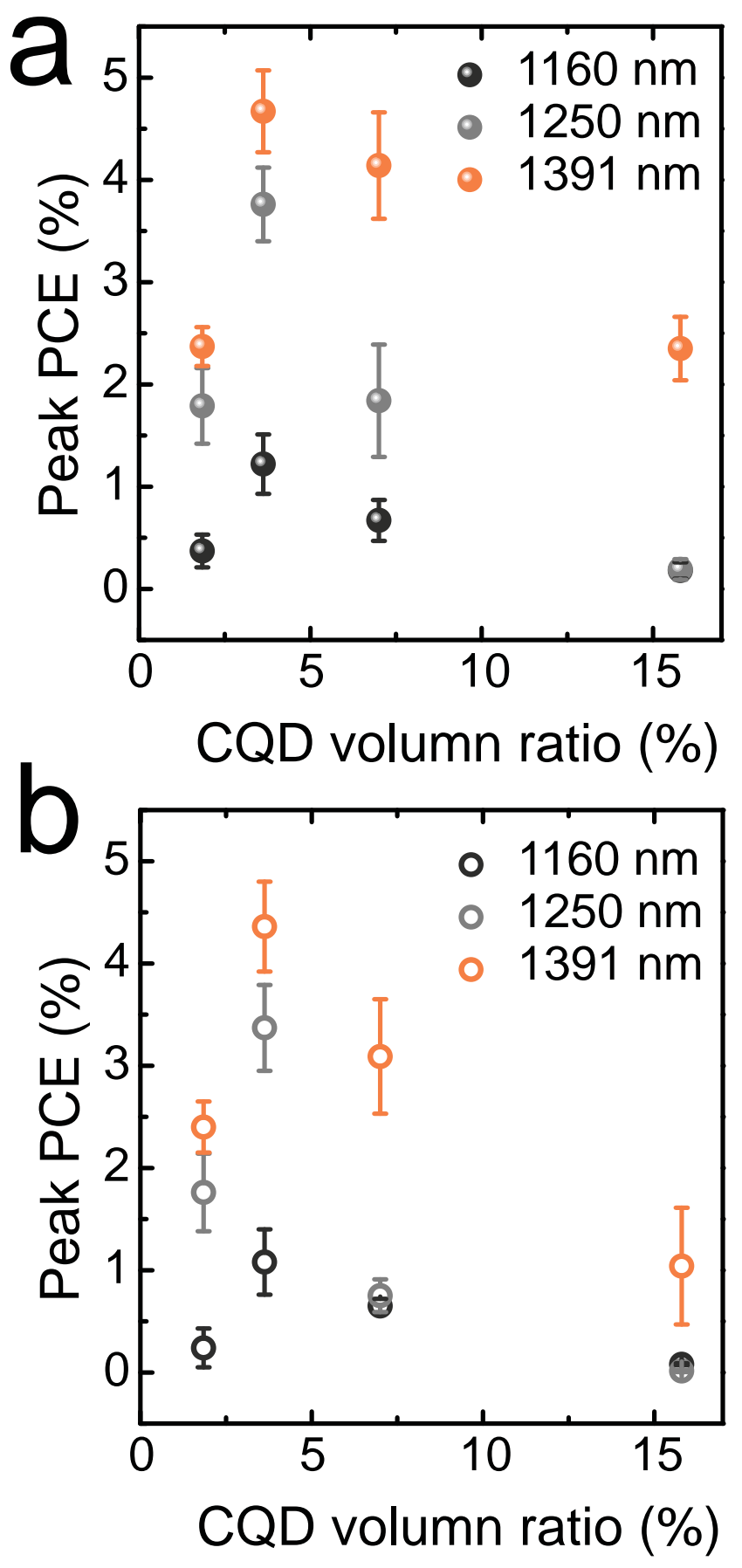


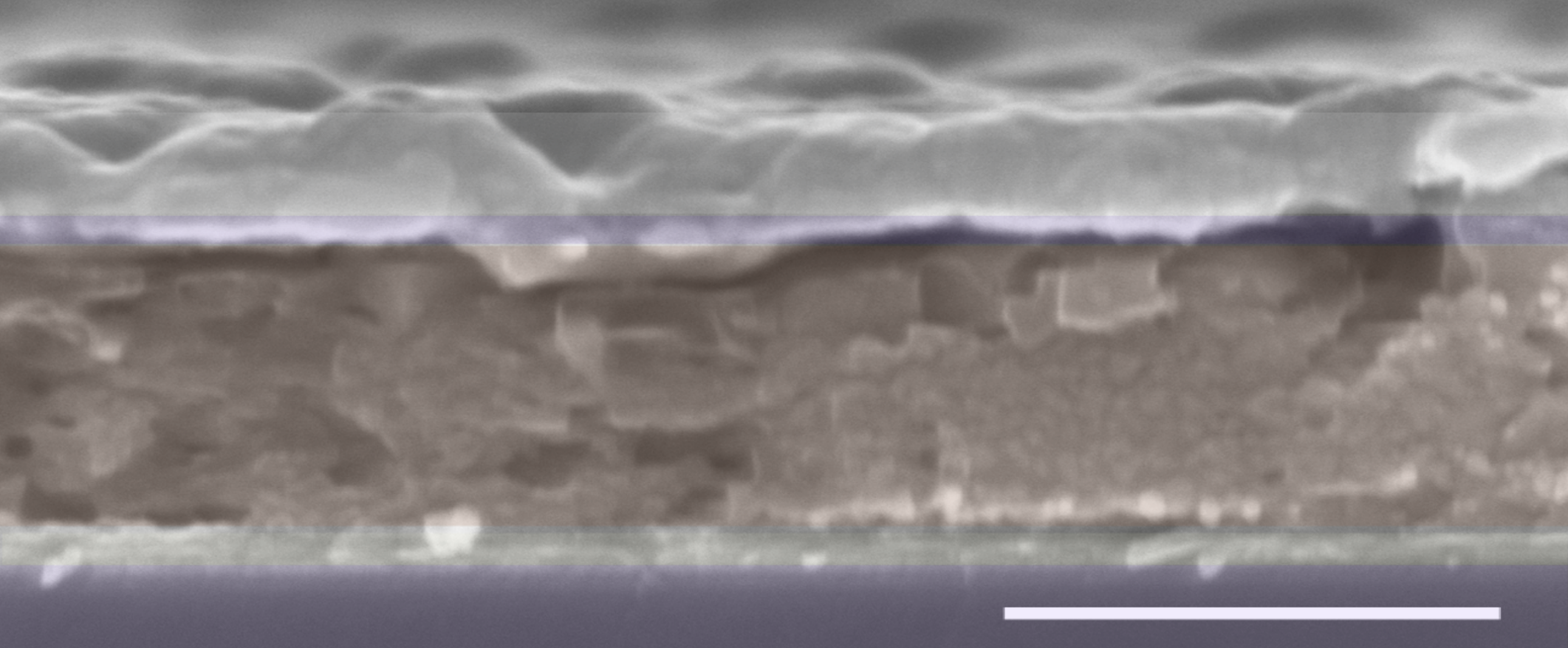

$500 \mathrm{~nm}$ 